\begin{tabular}{|c|l|}
\hline Title & Double network gels as polyelectrolyte gels with salt-insensitive swelling properties \\
\hline Author(s) & Nakajima, Tasuku; Chida, Takaharu; Mito, Kei; Kurokawa, Takay uki; Gong, Jian Ping \\
\hline Citation & $\begin{array}{l}\text { Soft matter, 16(23), 5487-5496 } \\
\text { https://doi.org/10.1039/dOsm00605j }\end{array}$ \\
\hline Issue Date & 2020-06-21 \\
\hline Doc URL & http://hdl.handle.net/2115/81939 \\
\hline Type & article(author version) \\
\hline File Information & chida_submission3_nohighlight.pdf \\
\hline
\end{tabular}

Instructions for use 


\section{Double-network gels as polyelectrolyte gels with salt-insensitive swelling properties}

Received 00th January 20xx, Accepted 00th January 20xx DOI: $10.1039 / \times 0 \times x 00000 x$

\begin{abstract}
Tasuku Nakajima*a,b,c, Takaharu Chidad ${ }^{d}$ Kei Mito ${ }^{d}$, Takayuki Kurokawaa,c and Jian Ping Gong a,b,c
Polyelectrolyte gels exhibit intrinsic salt-sensitive swelling behaviour, which causes size instability in ionic environments. Thus, polyelectrolyte gels that show salt-insensitive swelling have been anticipated for the applications in ionic environments, such as medical materials used in vivo. We found that double-network (DN) gels consisting of both a polyelectrolyte network and a non-ionic network are resistant to the salt-sensitive swelling. This resistance is attributed to their lower osmotic pressure originating from mobile ions relative to the osmotic pressure of mixing at swelling equilibrium. Our investigation indicated that the two contrasting network structures within DN gels are vital for achieving these properties, where the structures include a highly prestretched and sparse polyelectrolyte network and a coiled and dense non-ionic network. The salt-insensitivity of the DN gels has led to their unique applications in ionic environments.
\end{abstract}

\section{Introduction}

Swelling is a complex properties of polymer gels, where gels can swell to different extents in a solvent or solution depending on the several factors, such as affinity between polymer and solvent or ionic strength of the solution. ${ }^{1,2}$ One may take advantage of this environmentally dependent swelling when developing stimuli-responsive gels, where applications include sensors, ${ }^{3}$ drug delivery systems, ${ }^{1,4,5}$ and actuators. ${ }^{6}$ However, these properties are associated with unfavourable environmental stability.

The extent of swelling at equilibrium is determined by the balance of osmotic pressure and elastic pressure. ${ }^{7}$ Swelling in non-ionic polymer (NP) gels is promoted by the osmotic pressure that originates from the polymer-solvent mixing and is suppressed by the elastic pressure of the network. Polyelectrolyte (PE) gels contain fixed charges on polymer chains with corresponding counterions; thus, the osmotic pressure originating from the mobile ions should also be considered as a factor. ${ }^{7}$ A gel reaches equilibrium swelling when these pressures are balanced. PE gels typically exhibit a significantly larger swelling ratio than NP gels in a pure water or polar solvent due to higher osmotic pressure caused by the increased ionicity. At the same time, PE gels exhibit ionic strength-sensitive swelling behaviour. The swelling ratio of the PE gel decreases substantially with an increase in environmental

\footnotetext{
a. Faculty of Advanced Life Science, Hokkaido University, N21W11, Kita-ku, Sapporo, Japan. Tel\&Fax: +81-11-706-9016; E-mail: tasuku@sci.hokudai.ac.jp b. WPI-ICReDD, Hokkaido University, N21W10, Kita-ku, Sapporo, Japan. c. GI-CoRE, Hokkaido University, N21W11, Kita-ku, Sapporo, Japan

d. Graduate School of Life Science, Hokkaido University, N10W8, Kita-ku, Sapporo, Japan.

Electronic Supplementary Information (ESI) available: [The stress-deformation ratio curves of the PAMPS gels and the PAAm gels after swelling in the $\mathrm{NaCl}$ solutions]. See DOI: 10.1039/x0xx00000x
}

ionic strength because of a decrease in the concentration difference of mobile ions between gels in their environment. ${ }^{8-}$ ${ }^{11}$ Not only homo-PE gels but also copolymer gels consisting of $\mathrm{PE}$ and NP, and interpenetrating network (IPN) gels containing $\mathrm{PE}$ component exhibit salt-sensitive swelling behaviour. ${ }^{12-14}$ This can lead to unfavourable dimensional and mechanical instability of PE gels in ionic environments, including in the human body. Managing these changes in size and mechanical properties is required to allow for wider application of PE gels and their derivatives.

Double-network (DN) hydrogels have become popular due to their extreme toughness despite a high water content (ca. 90 wt.\%). ${ }^{15,16}$ Chemically cross-linked DN gels typically comprise of a PE first network and a non-ionic second network, and are referred to as PE/NP DN gels in this paper. PE/NP DN gels are usually synthesised as shown in Figure 1a. A densely crosslinked PE gel is prepared and immersed in a NP gel precursor solution. The PE gel becomes very swollen in the precursor solution due to its polyelectrolyte nature, leading to prestretching of the PE gel network strands. A sparsely cross-linked NP network forms within the swollen PE network to produce a $\mathrm{PE} / \mathrm{NP} \mathrm{DN}$ gel. These two networks have contrasting characteristics, where the PE network is rigid and brittle due to pre-stretching of the network strands and dense cross-linking, while the NP network is soft and stretchable due to its coiled network strands and sparse cross-linking. Optimised PE/NP DN gels can exhibit extreme fracture energies ranging 1,000 to $4,000 \mathrm{~J} \mathrm{~m}^{-2}$, while single PE and NP gels usually range 1 to $100 \mathrm{~J}$ $\mathrm{m}^{-2} \cdot{ }^{16,17}$ This high toughness of DN gels has been attributed to the contrasting double-network structure. ${ }^{18,19}$

Although the swelling properties of PE gels are typically sensitive to ionic strength of the environment, previous studies have indicated that the swelling behaviour of PE/NP DN gels is not sensitive to the ionic strength. ${ }^{20-22}$ Thus, despite having $P E$ as the first network, PE/NP DN gels do not behave like regular 
PE gels. This is a great advantage that allows for various unique applications of PE/NP DN gels in ionic environments. PE/NP DN gels have been used as a substrate for in vivo cartilage regeneration, where the DN gel embedded at the cartilage defect induced spontaneous cartilage regeneration. This success was attributed to the salt-insensitive polyelectrolyte nature of the DN gel. The PE component in the PE/NP DN gels played a vital role in activating the cartilage regeneration signalling pathway, ${ }^{23}$ while the ionic strength-insensitive swelling led to size stability of the gels embedded in vivo. ${ }^{22}$ $\mathrm{PE} / \mathrm{NP} \mathrm{DN}$ gels have also been used as a template for biomineralization, which involves the synthesis of minerals in/on materials under mild conditions to develop functional hybrid materials. ${ }^{24,25}$ PE gels show potential for use as a biomineralisation template because the ionic groups in the PE gels can serve as nucleation sites for crystal growth. ${ }^{26}$ However, PE gels shrink substantially when immersed in biomineralisation precursor salt solutions, which leads to high internal stress and macroscopic failure of the gel. PE/NP DN gels are a viable alternative for biomineralisation that offer nucleation sites due to the PE component and do not shrink significantly in salt solutions due to their salt-insensitive swelling ability. ${ }^{20,21}$ Furthermore, PE/NP DN gels may be used in marine environments, especially as an anti-fouling material against barnacles. ${ }^{27}$

This study aimed to investigate the basis of the ionic strength-insensitive swelling properties of PE/NP DN gels. The swelling behaviour of PE/NP DN gels in ionic environments were evaluated experimentally and theoretically. DN gel and its components were subjected to a swelling test in aqueous sodium chloride ( $\mathrm{NaCl}$ ) solutions (monovalent ions) and multivalent salt solutions. The experimental data and established theoretical knowledge were used to discuss the origin of PE/NP DN gel's salt-insensitive swelling behaviour. A model system was developed based on a typical DN gel consisting of poly(2-acrylamido-2-methylpropanesulfonic acid) (PAMPS) as the PE first network and polyacrylamide (PAAm) as the NP second network. This DN gel has been used widely in previous studies. ${ }^{16}$

The following symbols, superscripts and subscripts are used throughout this paper to describe the parameters of each gel component in different swelling states:

\section{Symbols:}

$\mathrm{m}$ : mass

w: weight fraction

$\phi$ : volume fraction

$\rho$ : density

Superscripts: state of gels

$\mathrm{p}$ : as-prepared state (before swelling)

w: equilibrium swelling state in water

s: equilibrium swelling state in salt solutions

d: dried state

No subscript: general case

Subscripts: components

1: PAMPS

2: PAAm

\section{s: solvent}

No subscript: total polymer (general case)

For example, $w_{1}^{\mathrm{s}}$ is the weight fraction of the PAMPS component at swelling equilibrium in a salt solution and $\phi_{2}^{\mathrm{d}}$ is the volume fraction of the PAAm component at the dried state. Schematic illustrations of the PAMPS. PAAm and DN gels at their as-prepared state and at equilibrium swelling in pure water are given in Figure $1 \mathrm{~b}$.

(a)

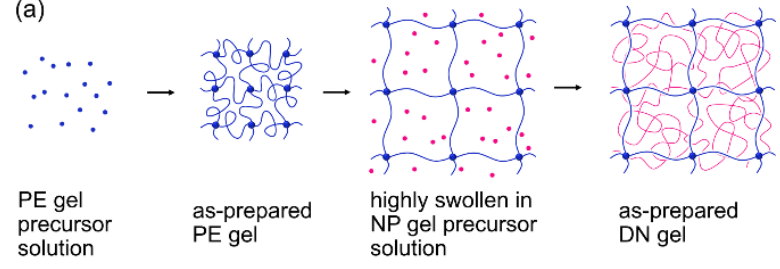

\begin{tabular}{llll} 
(b) & $\begin{array}{l}\text { PAMPS gel } \\
\text { (polyelectrolyte) }\end{array}$ & $\begin{array}{l}\text { PAAm gel } \\
\text { (non-ionic) }\end{array}$ & $\begin{array}{l}\text { PAMPS/PAAm } \\
\text { DN gel }\end{array}$ \\
\hline
\end{tabular}

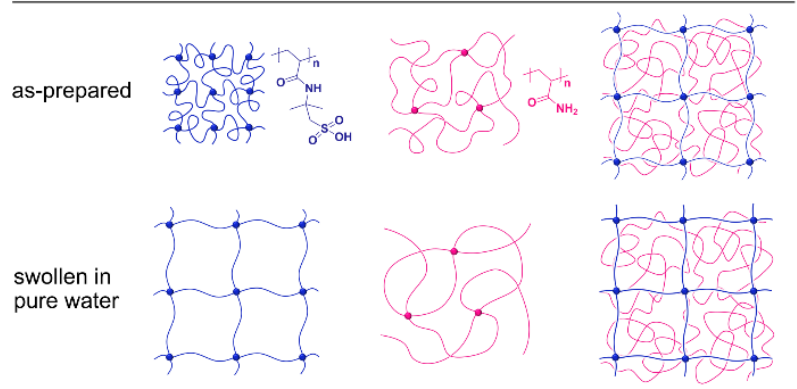

Figure 1. (a) Preparation of PE/NP DN gels consisting of a prestretched polyelectrolyte first network and a dense non-ionic second network. (b) The PAMPS, PAAm and PAMPS/PAAm DN gels at their as-prepared state and equilibrium swelling state in pure water, where the $D N$ gels consist of a prestretched PAMPS network and the coiled PAAm network.

\section{Theory}

A DN gel consists of three components, namely the first network, the second network and solvent. According to the Flory-Rehnerlike swelling theories, swelling equilibrium of a gel is determined by a balance of three independent contributions: $\Pi_{\mathrm{el}}, \Pi_{\mathrm{mix}}$ and $\Pi_{\mathrm{ion}} \cdot{ }^{7} \Pi_{\mathrm{el}}$ is the elastic pressure of the network that suppresses swelling. $\Pi_{\text {mix }}$ and $\Pi_{\text {ion }}$ are the osmotic pressure differences between a gel and its environment, that basically promote swelling, originating from the mixing of polymer and solvent and from mobile ions, respectively. At the swelling equilibrium, the osmotic pressure difference of a gel is balanced by its elastic pressure, giving Eq. 1 :

$\Pi_{\mathrm{el}}=\Pi_{\mathrm{mix}}+\Pi_{\mathrm{ion}}$

For simplicity, in this paper, $\Pi_{\mathrm{el}}, \Pi_{\mathrm{mix}}$ and $\Pi_{\text {ion }}$ are termed elastic, mixing and ionic contributions, respectively.

$\Pi_{\text {mix }}$ is often estimated using the Flory-Huggins lattice model. ${ }^{7}$ Assuming that no volume change occurs upon mixing the polymer networks and solvent, the change in Helmholtz's 
free energy during the formation of the ternary system by mixing two polymers and one solvent is given in Eq. 2:28

$\frac{\Delta F_{\text {mix }}}{n k T}=\frac{\phi_{1}}{N_{1}} \ln \phi_{1}+\frac{\phi_{2}}{N_{2}} \ln \phi_{2}+\phi_{s} \ln \phi_{s}+\chi_{12} \phi_{1} \phi_{2}+\chi_{1 s} \phi_{1} \phi_{s}+$ $\chi_{2 s} \phi_{2} \phi_{s}$

The volume of each lattice $\left(v_{\mathrm{S}}\right)$ was based on one solvent molecule, namely water. $v_{\mathrm{s}}=3 \times 10^{-29} \mathrm{~m}^{3}$ was estimated from the density of liquid water. ${ }^{29} n$ is total number of lattices in the gel, where the total volume of the gel $(V)=n v_{\mathrm{s}} . \phi_{i}$ is the volume fraction of the component $i$ in a gel, $N_{i}$ is number of lattices occupied by a single molecule of $i$, and $\chi_{i j}$ is Flory interaction parameter between the components $i$ and $j$, where $i$ and $j=1$, 2 or $s$ indicate the first network, second network, and solvent, respectively. Polymer networks have a quasi-infinite polymerisation degree $(N \gg 1)$, and the first two terms in Eq. 2 may be ignored for full-IPN gels, including DN gels, giving Eq. 3 :

$\frac{\Delta F_{\text {mix }}}{n k T} \cong \phi_{s} \ln \phi_{s}+\chi_{12} \phi_{1} \phi_{2}+\chi_{1 s} \phi_{1} \phi_{s}+\chi_{2 s} \phi_{2} \phi_{s}$

$\Pi_{\text {mix }}$ can be calculated by differentiating $F_{\text {mix }}$ with respect to $V$. The change in $V$ upon swelling of gels is caused by solvent migration, thus

$\mathrm{d} V=v_{s} \mathrm{~d} n_{s}$

where $n_{\mathrm{s}}$ is number of lattices occupied by the solvent. Eq. 5 is based on this relationship:

$\Pi_{\text {mix }}=-\frac{1}{v_{s}} \frac{\delta F_{\text {mix }}}{\delta n_{s}} \cong-\frac{k T}{V_{s}}\left(\ln \phi_{s}+\phi_{1}+\phi_{2}-\chi_{12} \phi_{1} \phi_{2}+\right.$

$\left.\chi_{1 s} \phi_{1}\left(1-\phi_{s}\right)+\chi_{2 s} \phi_{2}\left(1-\phi_{s}\right)\right)$

For $\Pi_{\mathrm{el}}$, we adopt the generalized 8-chain model network that shows affine deformation and has isotropic network strands. We also assume that elastic energy of the network is simply a sum of that of its network strands. Under these assumptions, $\Pi_{\mathrm{el}}$ can be estimated directly from the measured moduli of the gel without assuming Gaussian chain model:

$\Pi_{\mathrm{el}} \cong-G=-\frac{E}{3}$

where $G$ is the shear modulus and $E$ is the Young's modulus. ${ }^{7}$ Detailed derivation process of Eq. 6 is shown in Supplementary information. Note that many previous papers have estimated $\Pi_{\mathrm{el}}$ based on the classical rubber elasticity theory, which assumes Gaussian end-to-end distance distribution of the network strands. ${ }^{30}$ This estimation is valid for moderately swollen gels but is not applicable in highly swollen gels, including the PAMPS network used in this study. The actual elastic contribution of highly swollen gels is significantly larger than the classical estimation due to non-linear elasticity of the highly-prestretched network strands. ${ }^{31,32}$

For $\Pi_{\text {ion }}$, several factors that are related to charges contribute to the osmotic pressure of PE gels, including concentration differences of mobile ions between the gels and their environment and electrostatic repulsion between fixed changes on the network. ${ }^{33}$ Following the previous theoretical studies about swelling of PE gels, ${ }^{34,35}$ we only considered the concentration difference of mobile ions, although the other factors may affect slightly. When PAMPS is immersed in a $\mathrm{NaCl}$ solution, three mobile ion species should be present in the system, namely $\mathrm{Na}^{+}$and $\mathrm{Cl}^{-}$(from $\mathrm{NaCl}$ ) and $\mathrm{H}^{+}$(from PAMPS). Thus, $\Pi_{\text {ion }}$ of the gel in this study was:

$\Pi_{\text {ion }}=k T\left(\left(c_{H^{+}}^{g}+c_{N a^{+}}^{g}+c_{C l^{-}}^{g}\right)-\left(c_{H^{+}}^{e n v}+c_{N a^{+}}^{e n v}+c_{C l^{-}}^{e n v}\right)\right)$

where $c_{k}^{g}$ and $c_{k}^{e n v}$ are the concentration of mobile $k$ ions in the gel and environment, respectively. ${ }^{34}$ At swelling equilibrium, the system reaches Donnan equilibrium, where:

$\left(c_{H^{+}}^{g}+c_{N a^{+}}^{g}\right) c_{C l^{-}}^{g}=\left(c_{H^{+}}^{e n v}+c_{N a^{+}}^{e n v}\right) c_{C l^{-}}^{e n v}$

The volume of the environmental solution (containing $\mathrm{NaCl}$ ) is assumed to be infinite, thus:

$c_{H^{+}}^{e n v} \ll c_{N a^{+}}^{e n v}$

and

$c_{H^{+}}^{e n v}+c_{N a^{+}}^{e n v}=c_{C l^{-}}^{e n v} \cong c_{N a C l}$

where $c_{\mathrm{NaCl}}$ is the concentration of $\mathrm{NaCl}$ in the environment. Due to the electroneutrality of the gels:

$c_{H^{+}}^{g}+c_{N a^{+}}^{g}=c_{C l^{-}}^{g}+\frac{\alpha \phi_{1}}{v_{1}}$

where $v_{1}$ is the volume of a monomeric unit of PAMPS, estimated as $2 \times 10^{-28} \mathrm{~m}^{3} .{ }^{29} \alpha$ is the ratio of the mobile counterions to the total counterions in the PAMPS network, while other immobile counterions may be bound to the network (counterion condensation). ${ }^{36}$ Combining Eqs. 7, 8b and 9 gives Eq. 11:

$\left(c_{\mathrm{H}^{+}}^{g}+c_{N a^{+}}^{g}+c_{C l^{-}}^{g}\right)^{2} \cong \frac{\alpha^{2} \phi_{1}{ }^{2}}{V_{1}^{2}}+4 c_{\mathrm{NaCl}^{2}}$

$\Pi_{\text {ion }}$ can be calculated by substituting Eqs. $9 \mathrm{~b}$ and 11 in Eq. 7 , giving Eq. 12:

$\Pi_{\mathrm{ion}} \cong k T\left(\sqrt{\frac{\alpha^{2} \phi_{1}^{2}}{v_{1}^{2}}+4 c_{\mathrm{NaCl}}^{2}}-2 c_{\mathrm{NaCl}}\right)$

Substituting Eqs. 5, 6 and 12 in Eq. 1 gives the equation of state of the PE/NP DN gels at swelling equilibrium (Eq. 13):

$\frac{E}{3} \cong-\frac{k T}{v_{s}}\left(\ln \phi_{s}+\phi_{1}+\phi_{2}-\chi_{12} \phi_{1} \phi_{2}+\chi_{1 s} \phi_{1}\left(1-\phi_{s}\right)+\right.$
$\left.\chi_{2 s} \phi_{2}\left(1-\phi_{s}\right)\right)+k T\left(\sqrt{\frac{\alpha^{2} \phi_{1}^{2}}{v_{1}^{2}}+4 c_{\mathrm{NaCl}}^{2}}-2 c_{\mathrm{NaCl}}\right)$

\section{Experimental}

\section{Materials}

Acrylamide (AAm, Junsei Chemical Co., Ltd.) was recrystallised from chloroform. $N, N^{\prime}$-Methylenebisacrylamide (MBAA, Wako Pure Chemical Industries, Ltd.) was recrystallised from ethanol. 2-Acrylamido-2-methylpropanesulfonic acid (AMPS) was acquired from Toa Gosei Co., Ltd. 2-oxoglutaric acid, sodium chloride, calcium chloride, barium chloride, magnesium chloride, cobalt(II) chloride, nickel(II) chloride, iron(III) chloride 
were acquired from Wako Pure Chemical Industries, Ltd. and used as received.

\section{Preparation of gels}

Synthesis of PAMPS gels. 1 M AMPS, $40 \mathrm{mM} \mathrm{MBAA}$ and $10 \mathrm{mM}$ 2-oxogultaric acid were dissolved in pure water. The solution was transferred to an argon atmosphere and poured into a glass mould consisting of two soda-lime glass plates (thickness $=3 \mathrm{~mm}$ ) separated by a U-shaped silicone rubber sheet. The mould was exposed to UV radiation ( $365 \mathrm{~nm} ; 4 \mathrm{~mW} \mathrm{~cm}^{-2}$ ) for 6 h.

Synthesis of PAAm gels. 2 M AAm, 2 mM MBAA and 2 mM 2oxogultaric acid were dissolved in pure water. The solution was transferred to a glass mould in argon atmosphere as described above. The mould was irradiated under the same UV conditions for $8 \mathrm{~h}$.

Synthesis of DN gels. The PAMPS gel was immersed in an aqueous solution of $2 \mathrm{M} \mathrm{AAm}, 0.4 \mathrm{mM} \mathrm{MBAA}$ and $0.2 \mathrm{mM} 2-$ oxogultaric acid (PAAm precursor) for a minimum of 2 days. The PAMPS gel swelled significantly and was sandwiched by two glass plates and covered by a plastic wrap in an argon atmosphere. The gels were irradiated under the same UV conditions for $8 \mathrm{~h}$ to synthesise the PAAm network within the PAMPS gel.

\section{Swelling test}

Swelling test in water. The PAMPS gel, PAAm gel and DN gel were soaked in pure water at $297 \mathrm{~K}$ until swelling equilibrium was reached. The initial volume swelling ratio of the gels was defined as $\boldsymbol{V}^{\mathbf{w}} / \boldsymbol{V}^{\mathbf{p}}$, where $\boldsymbol{V}^{\mathbf{w}}$ is volume of the swollen gel in water and $\boldsymbol{V}^{\mathbf{p}}$ is volume of the as-prepared gel.

Swelling test in salt solutions. After swelling in pure water, the PAMPS gel, PAAm gel and DN gel were transferred to two series of aqueous salt solutions at $297 \mathrm{~K}$. The first was a $\mathrm{NaCl}$ series with varied concentrations $\left(\boldsymbol{c}_{\mathrm{NaCl}}\right)$ ranging 0 to $4 \mathrm{M}$, including the physiological concentration of $0.14 \mathrm{M}$. The second was a variety of chloride and multivalent countercation solutions, namely $\mathrm{CaCl}_{2}, \mathrm{MgCl}_{2}, \mathrm{BaCl}_{2}, \mathrm{CoCl}_{2}, \mathrm{NiCl}_{2}$ and $\mathrm{FeCl}_{3}$. The concentration of these multivalent salts was $1 \mathrm{M}$, regardless of cation valency. The volume de-swelling ratio of the gels was based on equilibrium swelling in water and is defined as $\boldsymbol{V}^{\mathbf{s}} / \boldsymbol{V}^{\mathbf{w}}$, where $\boldsymbol{V}^{\mathbf{s}}$ is the volume of the swollen gel in the salt solution.

\section{Estimation of polymer volume fraction}

Polymer volume fractions of the gel before swelling was estimated based on the weight fraction and density of each component. Polymer weight fraction of the gel was calculated based on its masses at the as-prepared state and the dried state:

$w^{\mathrm{p}}=\frac{m^{\mathrm{d}}}{m^{\mathrm{p}}}$
The weight ratio of the PAMPS and PAAm components in DN gels has been reported as 1:7. ${ }^{37}$ Thus, weight fraction of the PAMPS and PAAm components in the DN gels at the asprepared state can be calculated separately:

$w_{1}^{\mathrm{p}}=\frac{1}{8} w^{\mathrm{p}}$

$w_{2}^{\mathrm{p}}=w^{\mathrm{p}}-w_{1}^{\mathrm{p}}=\frac{7}{8} w^{\mathrm{p}}$

The corresponding polymer volume fraction of the gels at the as-prepared state $\left(\phi^{\mathrm{p}}\right)$ was estimated as:

$\phi^{\mathrm{p}}=\frac{\frac{w^{\mathrm{p}}}{\rho}}{\frac{w^{\mathrm{p}}}{\rho}+\frac{1-w^{\mathrm{p}}}{\rho_{S}}}$

where $\rho$ is the average density of the dried polymer and $\rho_{\mathrm{S}}$ is density of the solvent (water). It was assumed that no volume change occurred while mixing the polymers and solvent. The estimated $\rho$ values of PAMPS and PAAm were $1.739 \pm 0.3$ and $1.437 \pm 0.2 \mathrm{~g} \mathrm{~cm}^{-3}$, respectively, ${ }^{37,38}$ and the volume fraction of PAMPS $\left(\phi_{1}^{\mathrm{p}}\right)$ and PAAm $\left(\phi_{2}^{\mathrm{p}}\right)$ in the as-prepared DN gel were estimated separately based on these density values:

$\phi_{1}^{\mathrm{p}}=\frac{\frac{w_{1}^{\mathrm{p}}}{\rho_{1}}}{\frac{w_{1}^{\mathrm{p}}}{\rho_{1}}+\frac{w_{2}^{\mathrm{p}}}{\rho_{2}}+\frac{1-w^{\mathrm{p}}}{\rho_{S}}}$

$\phi_{2}^{\mathrm{p}}=\frac{\frac{w_{2}^{\mathrm{p}}}{\rho_{2}}}{\frac{w_{1}^{\mathrm{p}}}{\rho_{1}}+\frac{w_{2}^{\mathrm{p}}}{\rho_{2}}+\frac{1-w \mathrm{p}}{\rho_{s}}}$

where $\phi^{\mathrm{p}}$ of the DN gel is the sum of $\phi_{1}^{\mathrm{p}}$ and $\phi_{2}^{\mathrm{p}}$.

Based on $\phi^{\mathrm{p}}$, the polymer volume fraction of the gel at any swelling states $(\phi)$ was estimated as:

$\phi=\phi^{\mathrm{p}} \frac{V^{\mathrm{p}}}{V}$

Volume fraction of the PAMPS $\left(\phi_{1}\right)$ and PAAm $\left(\phi_{2}\right)$ in the DN gels was estimated similarly as:

$\phi_{1}=\phi_{1}^{\mathrm{p}} \frac{V^{\mathrm{p}}}{V}$

$\phi_{2}=\phi_{2}^{\mathrm{p}} \frac{V^{\mathrm{p}}}{V}$

The symbol $\phi_{1}$ will be also used for the volume fraction of PAMPS in the PAMPS gel, thus $\phi=\phi_{1}$ for the PAMPS gel.

\section{Mechanical characterizations.}

Uniaxial tensile and compression testing of the gels were performed at $297 \mathrm{~K}$. Nominal stress $(\sigma)$ is defined as the force divided by initial cross-sectional area of the sample. Deformation ratio $(\lambda)$ is defined as the length of the deformed sample divided by the undeformed length. Tensile tests were performed on DN gel and PAAm gel samples cut into dumbbell shapes (gauge length $=12 \mathrm{~mm}$ ) at a stretching rate of $0.14 \mathrm{~s}^{-1}$. The Young's modulus $(E)$ was estimated based on the initial slope of the stress-deformation ratio curve $(1<\lambda<1.1)$. Compression tests were performed on cylindrical PAMPS gel samples (diameter $=15 \mathrm{~mm}$; thickness $=2.3-3.8 \mathrm{~mm}$ ) using a TENSILON RTC-1310A compressive tester (Orientec Co.) at a 
compression rate of $0.1 \mathrm{~min}^{-1} . E$ was estimated based on the initial slope of the stress-deformation ratio curve $(0.9<\lambda<1)$. Note that $\lambda=1$ in the relaxed state and decreases with increasing compression.

\section{Results and Discussion}

\section{Swelling in water}

The initial volume swelling ratio of the PAMPS gel, PAAm gel and PAMPS/PAAm DN gel is given in Figure 2a. The PAMPS gel swelled significantly in pure water due to its ionic characteristics. The structure of the PAAm network in the PAAm and DN gels was very similar at their as-prepared states, while the DN gel included the additional prestretched PAMPS network (Figure 1b). The initial volume swelling ratio of the PAMPS/PAAm DN gel was smaller than the PAAm gel, despite containing the PAMPS network that exhibited a tendency toward substantial swelling.

The Young's modulus of the PAMPS gel and PAMPS/PAAm DN gel after swelling in pure water were similar, ranging 0.2 to $0.3 \mathrm{MPa}$, while that of the PAAm gel was much smaller (ca. 0.01 $\mathrm{MPa}$ ) (Figure 2b). The swollen PAMPS network was densely cross-linked and extremely prestretched. Thus, its elastic modulus was quite large despite its low polymer fraction. The swollen PAAm network was sparsely cross-linked and coiled. Thus, despite its high density, the PAAm gel was less stiff than the PAMPS gel.

The smaller initial volume swelling ratio of the DN gel than the PAAm gel is because their Young's modulus directly correlated to the elastic pressure (Eq. 6). The large Young's modulus of the DN gel indicated that the highly prestretched PAMPS network within the DN gel contributed to a large elastic pressure. This high elasticity counteracted the large mixing contribution, and the swelling of the DN gel was effectively suppressed by the stiff, highly prestretched PAMPS component.

\section{Swelling in salt solutions}

The volume de-swelling ratio and corresponding polymer volume fraction of the PAMPS gels, PAAm gels and PAMPS/PAAm DN gels were evaluated in $\mathrm{NaCl}$ aqueous solutions of various concentrations (Figures $3 a$ and $3 b$ ). The PAMPS gels shrank dramatically with increasing $\mathrm{NaCl}$ concentration, which is typical in a PE gel because the penetration of ions from the environment decreased the ionic contribution (Eq. 12). The PAAm gel did not shrink due to its non-ionic nature, while the DN gel similarly exhibited a swelling ratio that was not dependent on $\mathrm{NaCl}$ concentration. Even at the highest $\mathrm{NaCl}$ concentration of $4 \mathrm{M}$, the $\mathrm{DN}$ gel did not shrink significantly despite comprising a polyelectrolyte first network.

The de-swelling ratio of the PAMPS/PAAm DN gel immersed in various multivalent ion solutions demonstrated that the DN gel was resistant to changes in size in divalent or trivalent cation solutions (Figure $3 \mathrm{c}$ ). This salt-insensitive swelling provides strong evidence that the mixing contribution of the DN gel was much larger than the ionic contribution because the saltsensitive swelling characteristics of a PE gel is attributed to the
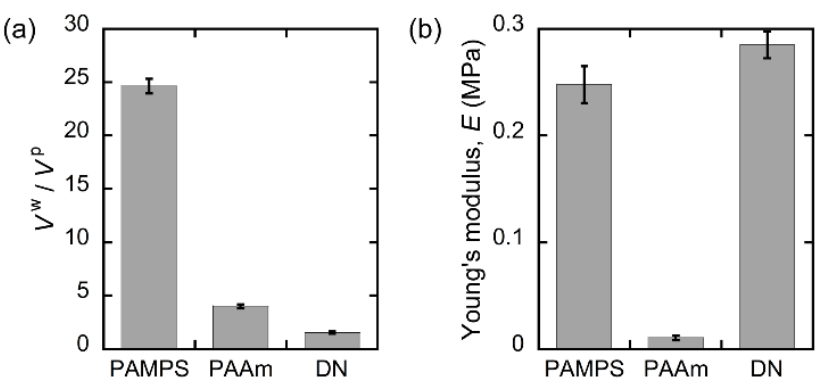

Figure 2. (a) Initial volume swelling ratios $\left(V^{\mathrm{w}} / V^{\mathrm{p}}\right)$ of the PAMPS, PAAm and PAMPS/PAAm DN gels, where $V^{\mathrm{p}}$ and $V^{\mathrm{w}}$ denote volume of a gel at as-prepared state and at equilibrium swelling state in pure water, respectively. (b) Young's modulus of the PAMPS, PAAm and PAMPS/PAAm DN gels after swelling in pure water.
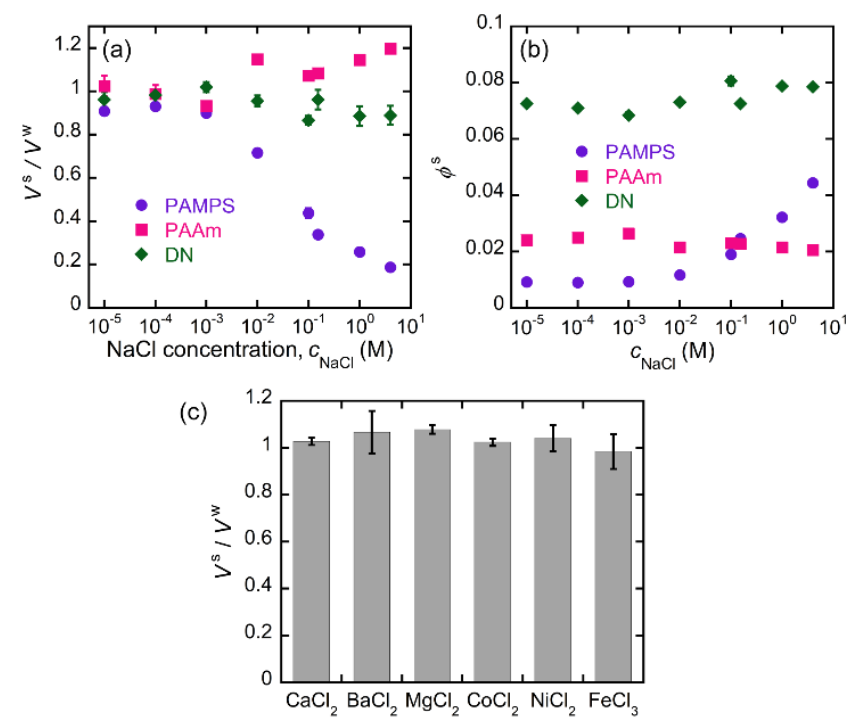

Figure 3. (a) De-swelling ratios $\left(V^{\mathrm{s}} / V^{\mathrm{w}}\right)$ of the PAMPS, PAAm and PAMPS/PAAm DN gels in aqueous $\mathrm{NaCl}$ solutions of various concentrations $\left(c_{\mathrm{NaCl}}\right)$, where $V^{\mathrm{S}}$ denotes volume of a gel at equilibrium swelling in the salt solution. (b) Polymer volume fraction at equilibrium swelling $\left(\phi^{\mathrm{s}}\right)$ of the gels in the $\mathrm{NaCl}$ aqueous solutions. (c) $V^{\mathrm{s}} / V^{\mathrm{w}}$ of the PAMPS/PAAm DN gel in various $1 \mathrm{M}$ multivalent salt aqueous solutions.

ionic one. The positive osmotic pressure of the DN gel was dominated by the dense non-ionic PAAm.

The Young's modulus of the PAMPS, PAAm and DN gels after swelling in $\mathrm{NaCl}$ solutions of various concentrations indicated that mechanical properties of the PAMPS gel was strongly influenced by $\mathrm{NaCl}$ concentration, while those of the PAAm and DN gels were minimally affected (Figure 4a). The Young's modulus and overall stress-deformation curves of the DN gels at the various $\mathrm{NaCl}$ concentrations almost identical (Figures $4 \mathrm{a}$ and $4 \mathrm{~b}$ ), indicating mechanical stability of the DN gels in ionic environments. The stress-deformation ratio curves of the PAMPS gels and the PAAm gels in the $\mathrm{NaCl}$ solution of various concentrations are shown in Figure S1 of Supplementary Information. The changes in mechanical behaviour of the gels in the presence of $\mathrm{NaCl}$ were directly related to the swelling behaviour under these conditions. Mechanical properties of an elastic gel are determined by its network structure. The significant shrinkage of the PAMPS gel in the $\mathrm{NaCl}$ solutions indicated that its mechanical properties were dependent on $\mathrm{NaCl}$ concentration, while the minimal effects on swelling of the 
PAAm and DN gels indicated that their mechanical properties were independent of $\mathrm{NaCl}$.

The relationship between swelling and the elastic properties of the gels was then investigated. The Young's modulus vs. the polymer volume fraction at equilibrium swelling $\left(E-\phi^{\mathrm{s}}\right)$ plots of the gels after swelling in the aqueous $\mathrm{NaCl}$ solutions are given in Figure 4c. The PAMPS gels exhibited a negative correlation between when $\phi^{\mathrm{s}}<0.02$, while the $E-\phi^{\mathrm{s}}$ relationships of the PAAm gel exhibited no clear trend due to the narrow polymer volume fraction range. The trends shown by the DN gels are also not clear because of the narrow $\phi^{\mathrm{s}}$ range, but a negative $E$ - $\phi^{\mathrm{s}}$ correlation could be found like the PAMPS gels. As the Young's modulus of the PAMPS/PAAm DN gel was mainly dominated by the fully stretched PAMPS, the $E$ of the PAMPS and DN gels should be both dependent on the volume fraction of PAMPS component in the PAMPS or DN gel $\left(\phi_{1}\right)$. Thus, the $E-\phi_{1}^{\mathrm{s}}$ relationship of the PAMPS and the DN gels was investigated (Figure 4d). As expected, data of both samples exhibited the single empirical power-law relationship $E \propto \phi_{1}^{-1.2}$, suggesting that the $E$ of the both gels was dominated by $\phi_{1}$.

Negative $E$ - $\phi$ correlation ( $E \propto \phi_{1}^{-1.2}$ ) is uncommon in gels The modulus of an elastic gel is determined by its network strand density $(v)$ and elastic energy per a network strand $(U)$ based on the relationship $E \sim v U$. Swelling (volume increase) of a gel results in a decreased $\phi$, causing a decrease in $v\left(v \propto \phi^{1}\right)$ and increase in $U$. Network strands characterised as Gaussian chains will behave like Hookean springs $\left(U \propto \phi^{-2 / 3}\right)$. These two relationships lead to a positive $E$ - $\phi$ correlation, i.e. $E \propto \phi^{1 / 3}$, which has been widely reported. The exponent of a real system may not be exactly $1 / 3$ and can vary slightly due to the condition of a gel. ${ }^{39,40}$ Conversely, highly stretched network strands exhibit non-linear strain hardening and an abrupt increase in elastic energy upon swelling, which is typically described by the inverse-Langevin function or the worm-like chain model ${ }^{41}$ This strong nonlinearity leads to large increases in $U$ with a decrease in $\phi$, and thus negative $E-\phi$ correlation. This behaviour has been observed in highly swollen PE gels. ${ }^{34}$ Thus, the negative $E$ $\phi^{\mathrm{s}}$ correlation of the PAMPS and DN gels means that the PAMPS network strands were highly stretched and possess a large elastic energy. ${ }^{32}$

\section{Discussion}

The experimental data confirmed that the PAMPS/PAAm DN gels exhibit salt-insensitive swelling and mechanical properties even at extremely high ionic strength. The salt-insensitivity of the PE/NP DN gels were then characterised using the theoretical estimations of the mixing and ionic contributions to the total swelling pressure of the gels. These estimations were based on the equation of state of the PE/NP DN gels (Eq. 13). The equation considers various parameters, where $T(297 \mathrm{~K})$ and $c_{\mathrm{NaCl}}$ were experimental conditions and $\phi_{1}^{\mathrm{s}}$ and $\phi_{2}^{\mathrm{s}}$ were experimentally determined. The remaining parameters $\chi_{12}, \chi_{1 s}$, $\chi_{2 s}$, and $\alpha$ were based on fitting parameters that were assumed to be independent of other parameters such as $\mathrm{NaCl}$ concentration. These experimental and fitted values were used to calculate the Young's modulus.
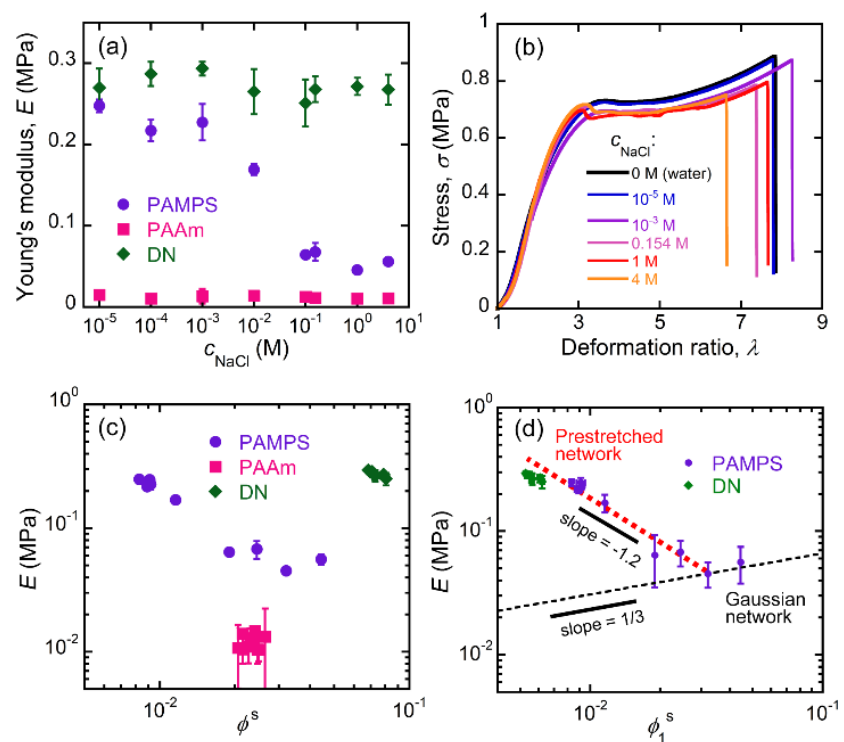

Figure 4. (a) Young's modulus ( $E$ ) of the PAMPS, PAAm and PAMPS/PAAm DN gels in aqueous $\mathrm{NaCl}$ solutions with varying environmental $\mathrm{NaCl}$ concentration $\left(c_{\mathrm{NaCl}}\right)$. (b) Nominal tensile stress-deformation ratio curves of the DN gels after swelling in the $\mathrm{NaCl}$ solutions. (c) Relationship between $E$ and polymer volume fraction at equilibrium swelling $\left(\phi^{\mathrm{s}}\right)$ of the PAMPS, PAAm and DN gels. (d) Relationship between $E$ and $\phi_{1}^{\text {s }}$ in the PAMPS and DN gels, where the empirical power-law relationship $E \propto \phi_{1}^{-1.2}$ is applicable at small $\phi_{1}^{s}$ values in a large swelling regime. The relationship of these gels deviated strongly from the typical $E \propto \phi^{1 / 3}$ based on a gel with Gaussian network strands.
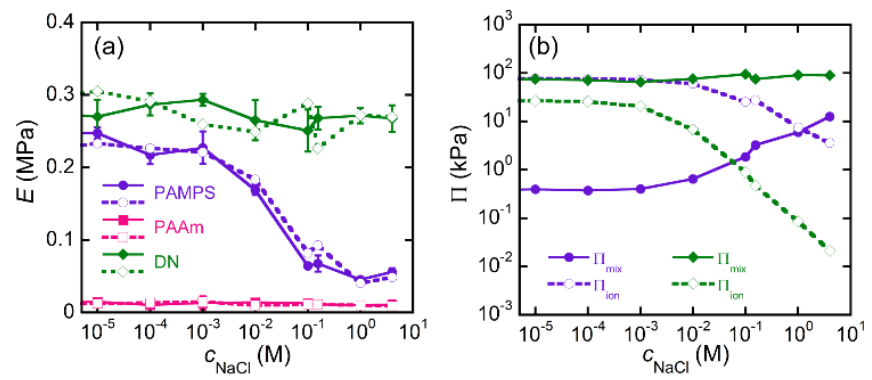

Figure 5. (a) Theoretical calculation of the relationship between $\mathrm{NaCl}$ concentration and Young's modulus $(E)$ of the PAMPS, PAAm and PAMPS/PAAm DN gels. The filled points denote the experimental values and the open points denote the calculated values (Eq. 13). The parameters best fit for the calculation are given in Table 1. (b) $\Pi_{\text {mix }}$ and $\Pi_{\text {ion }}$ of the PAMPS (purple circle) and the DN (green diamond) gels calculated based on Eqs. 5 and 12 .

Table 1. The best fitting parameters for each gel.
PAMPS gel
PAAm gel
DN gel

Parameter this work literature this work literature this work literature

\begin{tabular}{lcccccc}
$\chi_{12}$ & - & - & - & - & 0.0160 & $0.015^{18}$ \\
$\chi_{1 \mathrm{~s}}$ & 0.469 & $0.48^{28}$ & - & - & 0.299 & $0.3^{18}$ \\
$\chi_{2 \mathrm{~s}}$ & - & - & 0.456 & $0.48-0.51^{29}$ & 0.433 & $0.45^{18}$ \\
$\alpha$ & 0.413 & $0.35^{30}$ & - & - & 0.232 & - \\
\hline
\end{tabular}

The relationship between $E$ and $\mathrm{NaCl}$ concentration of the PAMPS, PAAm and DN gels based on Eq. 13 and the best fit parameters is shown in Figure $5 \mathrm{a}$, where the fitting parameters are given in Table 1 . The experimental and calculated results were very similar, although the calculated $E$ values exhibited slight deviates attributed to experimental errors in determining $\phi_{1}^{\mathrm{s}}$ and $\phi_{2}^{\mathrm{s}}$. The best fit values were closely related to the literature values. The $\chi_{12}, \chi_{1 s}$ and $\chi_{2 s}$ values of the 
PAMPS/PAAm DN gels in water were determined experimentally in a previous study as $0.3,0.45$ and 0.015 , respectively, using the neutron scattering technique, ${ }^{29}$ and closely reflect the best fit values of $0.299,0.433$ and 0.0160 , respectively. The $\chi_{1 s}$ and $\chi_{2 s}$ values of the PAMPS and PAAm gel in water were estimated to be 0.48 and $0.48-0.51$, respectively, based on the swelling test. ${ }^{12,42}$ These experimental values were close to the best fit values of 0.469 and 0.456 , respectively. The best fit ratio of the mobile counterions among total counterions $(\alpha)$ of the PAMPS gel was 0.413 , which is consistent with a reported value of $0.35 .{ }^{30}$ The best fit $\alpha$ of the DN gel was 0.232 , only $56 \%$ of the PAMPS gel value. This implies that counterion condensation was more obvious in the DN gel than the PAMPS gel. This phenomenon was attributed to the difference in relative permittivity between the PAMPS and the DN gels. According the condensation theory by Manning, the minimum charge spacing allowed of a polyelectrolyte chain is the Bjerrum length, $l_{B}$, which is defined as:

$l_{\mathrm{B}}=\frac{e^{2}}{\varepsilon k T}$

where $e$ is the charge of an electron and $\varepsilon$ is the relative permittivity. ${ }^{36}$ Counterion condensation occurs when the distance between two adjacent ionic groups $\left(l_{0}\right)$ is closer than $\left(l_{B}\right)$. Thus, $\alpha$ can be determined as

$\alpha=\frac{l_{0}}{l_{\mathrm{B}}}$

Eqs. 19 and 20 indicate that $\alpha$ is positively dependent on relative permittivity $(\alpha \propto \varepsilon)$. The dense PAAm component of the DN gel should result in a difference in relative permittivity $(\varepsilon)$ compared to the PAMPS gel. The best fit $\alpha$ value for the DN gel was $56 \%$ the PAMPS gel value, thus the $\varepsilon$ value of the DN gel was expected to be $56 \%$ of the PAMPS gel value. Assuming the $\varepsilon$ of the PAMPS gel was almost equal to that of pure water, namely 78 , the $\varepsilon$ of the DN gel was 44 (56\% of 78 ). Ishida et al. reported that the $\varepsilon$ value of poly( $\mathrm{N}$-isopropylacrylamide) hydrogels at room temperature was $46 .{ }^{44}$ Poly $(\mathrm{N}$ isopropylacrylamide) is a derivative of PAAm, indicating that an $\varepsilon$ value of 44 for the PAMPS/PAAm DN gels was reasonable.

As all of the best fit values were validated, the $\Pi_{\text {mix }}$ and $\Pi_{\text {ion }}$ of the gels were calculated based on Eqs. 5 and 12. The calculated $\Pi_{\text {mix }}$ and $\Pi_{\text {ion }}$ values of the PAMPS and DN gels were dependent on $\mathrm{NaCl}$ concentration and there was a clear difference between the two gels at a low $\mathrm{NaCl}$ concentration (Figure $5 b$ ). The $\Pi_{\text {ion }}$ value of the PAMPS gel was two or three orders of magnitude higher than $\Pi_{\text {mix }}$, while the $\Pi_{\text {ion }}$ value of the DN gels was significantly smaller than $\Pi_{\text {mix }}$. Thus, at low $\mathrm{NaCl}$ concentration, the swelling of the PAMPS gels was $\Pi_{\text {ion }}{ }^{-}$ dominant while the swelling of the DN gels was $\Pi_{\text {mix }}$-dominant. The large $\Pi_{\text {mix }}$ component in the DN gels was attributed to the dense PAAm. The $\Pi_{\text {ion }}$ value of both gels decreased with increasing $\mathrm{NaCl}$ concentration, as predicted by Eq. 12. The PAMPS gel exhibited $\Pi_{\text {ion }}$-dominant swelling and thus the swelling ratio decreased with increasing $\mathrm{NaCl}$ concentration, whereas the $\Pi_{\mathrm{mix}}$-dominant swelling with negligible effect of

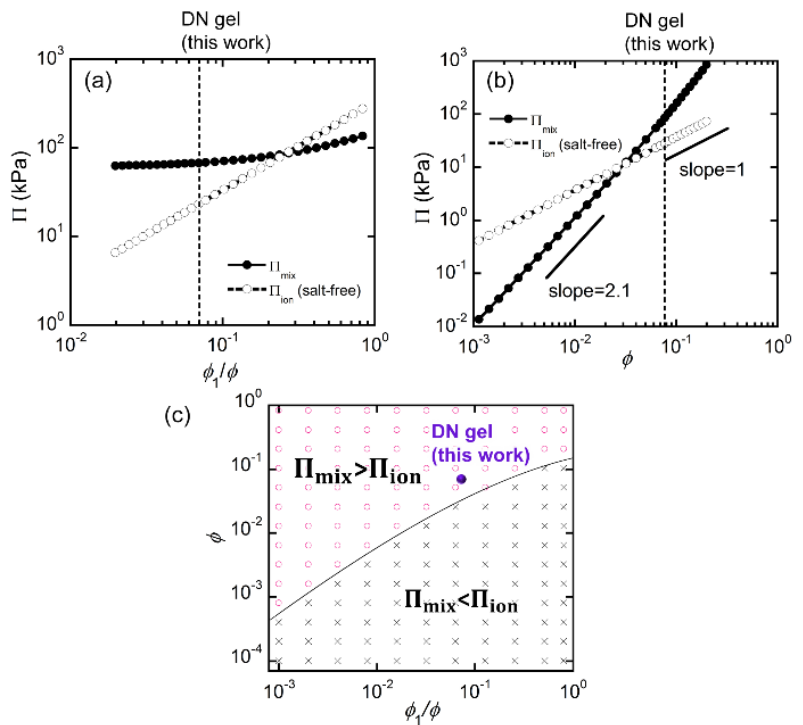

Figure 6. Magnitude correlation of calculated $\Pi_{\text {mix }}$ and $\Pi_{\text {ion }}$ of the PAMPS/PAAm DN gel. (a) Dependence of the $\Pi_{\text {mix }}$ and $\Pi_{\text {ion }}$ on $\phi_{1} / \phi$ with fixed $\phi=0.070$; (b) dependence of the $\Pi_{\text {mix }}$ and $\Pi_{\text {ion }}$ on $\phi$ with fixed $\phi_{1} / \phi=0.077$. (c) A phase diagram for the magnitude correlation of $\Pi_{\mathrm{mix}}$ and $\Pi_{\text {ion }}$ against $\phi_{1} / \phi$ and $\phi$. Circle magenta symbols denote $\Pi_{\text {mix }}>\Pi_{\text {ion }}$, while cross black symbols denote $\Pi_{\text {mix }}<\Pi_{\text {ion }}$. The data of the DN gel swollen in water, denoted as a purple filled circle, is in the former regime, corresponding to its salt-insensitive swelling property.

$\Pi_{\text {ion }}$ of the DN gel allowed for a swelling ratio independent of $\mathrm{NaCl}$ concentration.

As discussed above, the salt-insensitive swelling behaviour of the PAMPS/PAAm DN gels was attributed to a larger $\Pi_{\text {mix }}$ than $\Pi_{\text {ion }}$. The structural requirements of these properties were subsequently investigated by calculating $\Pi_{\text {mix }}$ and $\Pi_{\text {ion }}$ of the PAMPS/PAAm DN gels in salt-free conditions by changing two variables in Eqs. 5 and 12, namely $\phi_{1} / \phi$ (volume ratio of the PE among the total polymer in the gel) and $\phi$ (absolute polymer volume fraction in the gel). The $\Pi_{\text {mix }}$ and $\Pi_{\text {ion }}$ values of the DN gels were dependent on $\phi_{1} / \phi$ (Figure 6a). The $\Pi_{\text {ion }}$ value of the DN gel increased with increasing $\phi_{1} / \phi$, while the $\Pi_{\text {mix }}$ value remained constant. According to Eqs. 5 and 12, the volume fraction of the PAMPS $\left(\phi_{1}\right)$ contributed to both $\Pi_{\text {mix }}$ and $\Pi_{\text {ion }}$, while the volume fraction of the PAAm $\left(\phi_{2}\right)$ only contributed to $\Pi_{\mathrm{mix}}$. Thus, the $\phi_{1} / \phi$ ratio needs to be small enough to achieve a $\Pi_{\text {mix }}$ value larger than $\Pi_{\text {ion }}$. The $\Pi_{\text {mix }}$ and $\Pi_{\text {ion }}$ values of the DN gels were also dependent on $\phi$ (Figure 6b). Both $\Pi_{\text {mix }}$ and $\Pi_{\text {ion }}$ increased with increasing $\phi$ but exhibited significantly different scaling relationships of $\Pi_{\text {mix }} \propto$ $\phi^{2.1}$ and $\Pi_{\text {ion }} \propto \phi^{1}$. Note that $\Pi_{\text {mix }} \propto \phi^{2.1}$ is in good agreement with the well-known scaling relationship of $\Pi_{\text {mix }} \propto$ $\phi^{9 / 4}$ for polymers in their good solvent. ${ }^{41}$ Since $\Pi_{\text {mix }}$ is more sensitive to $\phi$ than $\Pi_{\text {ion }}$, it can be imagined that enough large $\phi$ is required to achieve $\Pi_{\mathrm{mix}}>\Pi_{\text {ion }}$. For example, if $\phi_{1} / \phi=0.07$, the lower limit of $\phi$ for $\Pi_{\text {mix }}>\Pi_{\text {ion }}$ is 0.03 . The phase diagram for $\Pi_{\text {mix }}>\Pi_{\text {ion }}$ shown in Figure $6 c$ exhibits that both of small $\phi_{1} / \phi$ and large $\phi$ are necessary to achieve $\Pi_{\text {mix }}>\Pi_{\text {ion }}$ and saltinsensitive swelling of the gels. Effect of the gel parameters (such as $\alpha$ ) on the phase diagram is shown in Figure S2 of Supplementary information. Also, the relationship between the 


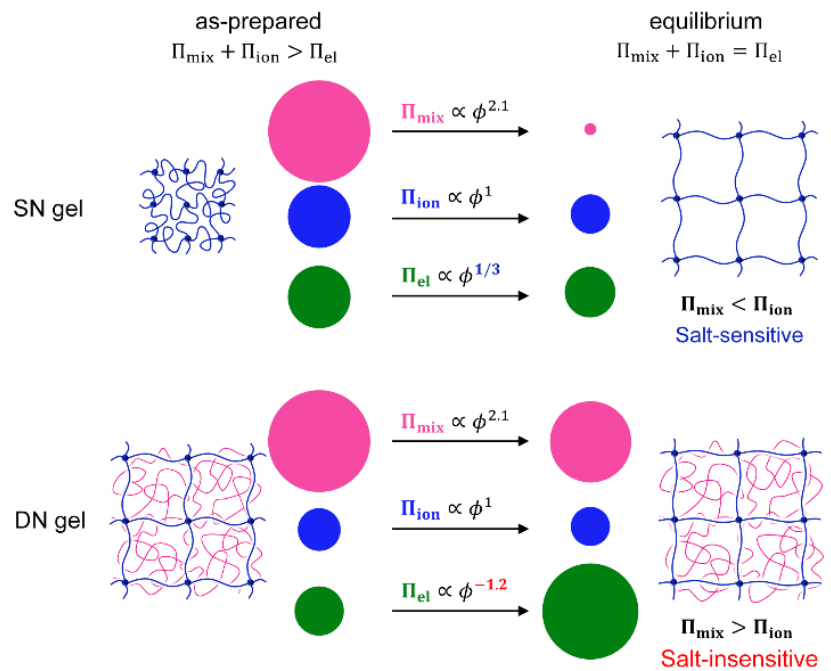

Figure 7. Schematic illustrations of change of the three contributions to the total swelling pressure of a polyelectrolyte SN gel and a DN gel upon swelling. Area of circles denote relative magnitude of the three contributions of the gels at each state.

toughening condition of DN gels and the phase diagram is discussed in Figure S3 of Supplementary information.

However, we should emphasize that it is quite difficult for a PE gel to achieve large $\phi$ at its equilibrium swelling state for which contrasting double-network structure is a requirement. Let us compare an as-prepared PE/NP DN gel and a singlenetwork (SN) PE gel whose polymer volume fractions at asprepared state, $\phi^{\mathrm{p}}$, are sufficiently large. As illustrated in Figure 7, the as-prepared DN gel has the prestretched PE first network, while the as-prepared SN gel has a coiled PE network. They can satisfy $\Pi_{\text {mix }}>\Pi_{\text {ion }}$ at their as-prepared state with enough large $\phi^{\mathrm{p}}$ (Figure 6c). Since these as-prepared gels should show $\Pi_{\mathrm{el}} \ll$ $\Pi_{\text {mix }}+\Pi_{\text {ion }}$ due to their large $\phi^{\mathrm{p}}$, the gels swell when immersed in water. In the case of the DN gel, because the prestretched first network shows negative $E-\phi$ relationship as shown in Figure $4 \mathrm{~d}$ (empirically $E \propto \phi^{-1.2}$ in this work) and sustains large elastic energy, swelling greatly increases their elastic contribution $\Pi_{\mathrm{el}}$. As a consequence, the large osmotic pressure $\Pi_{\text {mix }}+\Pi_{\text {ion }}$ of the DN gel can be balanced by $\Pi_{\mathrm{el}}$ just by a little swelling, and the DN gel can maintain large polymer fraction $\phi^{\mathrm{w}}$ even at its equilibrium swelling state in water. Thus, the DN gel still satisfy $\Pi_{\text {mix }}>\Pi_{\text {ion }}$ at their equilibrium swelling state, and the DN gel shows salt-insensitive swelling property. In the case of the SN gel, because its network strand is coiled at its as-prepared state, the gel initially shows positive $E-\phi$ relationship (typically $E \propto \phi^{1 / 3}$ ). It means swelling of the gel network leads to a decrease in its elastic contribution $\Pi_{\mathrm{el}}$. Thus, at the initial swelling stage, the elastic pressure of the network cannot suppress the its swelling. With proceeding of the swelling, all three contributions, $\Pi_{\mathrm{el}}, \Pi_{\mathrm{mix}}$, and $\Pi_{\mathrm{ion}}$, decrease with decrease of $\phi$ roughly as $\Pi_{\mathrm{el}} \propto \phi^{1 / 3}, \Pi_{\mathrm{mix}} \propto \phi^{2.1}$, and $\Pi_{\text {ion }} \propto \phi^{1}$. Because decays of $\Pi_{\text {mix }}$ and $\Pi_{\text {ion }}$ with decrease of $\phi$ are faster than $\Pi_{\mathrm{el}}$ and the $E-\phi$ relationship may become positive at low $\phi$, finally the three contributions are balanced at certain $\phi^{\mathrm{w}}$ after significant swelling. As a consequence, the resulting $\phi^{\mathrm{w}}$ of the SN gel is much smaller than the threshold $\phi$ for $\Pi_{\text {mix }}>\Pi_{\text {ion }}$, so that the obtained SN gel swollen in water shows salt-sensitive swelling property.

\section{Conclusions}

In conclusion, we confirmed that swelling and mechanical properties of the PAMPS/PAAm DN gels are certainly insensitive to concentration and valency of ions in the environment although the DN gels contain polyelectrolyte components. In the case of the DN gels, the mixing contribution $\Pi_{\mathrm{mix}}$ is superior to the ionic contribution $\Pi_{\text {ion }}$, which is the direct reason of saltinsensitive swelling behaviour of the DN gels. The highlyprestretched first network of the DN gels the key to achieve $\Pi_{\text {mix }}>\Pi_{\text {ion }}$ at their swelling equilibrium. This study has clarified that the contrasting double-network structure in DN gels not only works to improve mechanical properties of the gels but also brings unique salt-insensitive swelling ability. The combination of high toughness and salt insensitivity of the DN gels can lead various applications as already described in the introduction part, such as tough and stable polyelectrolyte gels that work in biological and marine environments, template for biomineralisation, and tough gels with large conductivity (by immersion in a salt solution) for electronics applications.

In addition, this work proposes a novel idea: the liberation of the swelling ratio of a gel from its characters. The swelling ratio of a polymer gel in a solvent is generally determined by characters of the polymer-solvent pair, including affinity between polymer and solvent and charges of the polymer; thus, they have been considered inseparable. However, once the contrasting PE/NP DN gel is formed, the swelling ratio of the first PE network in the DN gel is no longer controlled by characters of the PE network-solvent pair, but by those of the NP network-solvent pair. The independency of the swelling ratio from its characters may cause some unexpected phenomena, such as large swelling of the first network in its poor solvent. This can be realized by the immersion of a DN gel into the solvent that is poor solvent for the first target network but the good solvent for the second network.

\section{Conflicts of interest}

There are no conflicts to declare.

\section{Acknowledgements}

This research was supported by JSPS KAKENHI grant numbers 17H04891 and 17H06144. The Institute for Chemical Reaction Design and Discovery (WPI-ICReDD) was established by World Premier International Research Initiative (WPI), MEXT, Japan. T. N. thanks Ryuji Kiyama (Hokkaido University) for his useful comments on this manuscript.

\section{References}

1 N. A. Peppas and A. R. Khare, Adv. Drug Deliv. Rev., 1993, 11, $1-35$. 
2 M. Quesada-Pérez, J. A. Maroto-Centeno, J. Forcada and R. Hidalgo-Alvarez, Soft Matter, 2011, 7, 10536-10547.

3 I. Tokarev and S. Minko, Soft Matter, 2009, 5, 511-524.

4 Y. Qiu and K. Park, Adv. Drug Deliv. Rev., 2001, 53, 321-339.

5 T. R. Hoare and D. S. Kohane, Polymer, 2008, 49, 1993-2007.

6 L. Ionov, Adv. Funct. Mater., 2013, 23, 4555-4570.

7 P. J. Flory, Principles of Polymer Chemistry, Cornell University Press, Ithaca, NY, 1953.

8 A. Katchalsky, S. Lifson and H. Exsenberg, J. Polym. Sci., 1951, 7, 571-574.

9 A. Katchalsky and I. Michaeli, J. Polym. Sci., 1955, 15, 69-86.

10 R. Skouri, F. Schosseler, J. P. Munch and S. J. Candau, Macromolecules, 1995, 28, 197-210.

11 M. Rubinstein, R. H. Colby, A. V. Dobrynin and J.-F. Joanny, Macromolecules, 1996, 29, 398-406.

12 S. Durmaz and O. Okay, Polymer, 2000, 41, 3693-3704.

13 S. J. Kim, S. U. R. Shin, N. G. Kim and S. I. Kim, J. Macromol. Sci. - Pure Appl. Chem., 2005, 42 A, 1073-1083.

14 Q. Tang, J. Wu, J. Lin, Q. Li and S. Fan, J. Mater. Sci., 2008, 43, 5884-5890.

15 J. P. Gong, Y. Katsuyama, T. Kurokawa and Y. Osada, Adv. Mater., 2003, 15, 1155-1158.

16 J. P. Gong, Soft Matter, 2010, 6, 2583-2590.

17 Y. Tanaka, R. Kuwabara, Y. H. Na, T. Kurokawa, J. P. Gong and Y. Osada, J. Phys. Chem. B, 2005, 109, 11559-11562.

18 H. R. Brown, Macromolecules, 2007, 40, 3815-3818.

19 Y. Tanaka, Europhys. Lett., 2007, 78, 56005.

20 T. Nonoyama, S. Wada, R. Kiyama, N. Kitamura, M. T. I. Mredha, X. Zhang, T. Kurokawa, T. Nakajima, Y. Takagi, K. Yasuda and J. P. Gong, Adv. Mater., 2016, 28, 6740-6745.

21 R. Kiyama, T. Nonoyama, S. Wada, S. Semba, N. Kitamura, T. Nakajima, T. Kurokawa, K. Yasuda, S. Tanaka and J. P. Gong, Acta Biomater., 2018, 81, 60-69.

22 C. Azuma, K. Yasuda, Y. Tanabe, H. Taniguro, F. Kanaya, A. Nakayama, Y. M. Chen, J. P. Gong and Y. Osada, J. Biomed. Mater. Res. A, 2007, 81A, 373-380.

23 K. Goto, T. Kimura, N. Kitamura, S. Semba, Y. Ohmiya, S. Aburatani, S. Matsukura, M. Tsuda, T. Kurokawa, J. P. Gong, S. Tanaka and K. Yasuda, J. Biomed. Mater. Res. A, 2016, 104 734-746.

24 F. Nudelman and N. A. J. M. Sommerdijk, Angew. Chem. Int Ed., 2012, 51, 6582-6596.

25 A. Arakaki, K. Shimizu, M. Oda, T. Sakamoto, T. Nishimura and T. Kato, Org. Biomol. Chem., 2015, 13, 974-989.

26 T. Iwatsubo, R. Kishi, T. Miura, T. Ohzono and T. Yamaguchi, J. Phys. Chem. B, 2015, 119, 8793-8799.

27 T. Murosaki, T. Noguchi, K. Hashimoto, A. Kakugo, T. Kurokawa, J. Saito, Y. M. Chen, H. Furukawa and J. P. Gong, Biofouling, 2009, 25, 657-666.

28 C. C. Hsu and J. M. Prausnitz, Macromolecules, 1974, 7, 320324.

29 T. Tominaga, V. R. Tirumala, S. Lee, E. K. Lin, J. P. Gong and W.L. Wu, J. Phys. Chem. B, 2008, 112, 3903-3909.

30 H. M. James and E. Guth, J. Chem. Phys., 1943, 11, 455-481.

31 M. M. Ozmen and O. Okay, Polym. Bull., 2004, 52, 83-90.

32 K.-I. Hoshino, T. Nakajima, T. Matsuda, T. Sakai and J. P. Gong, Soft Matter, 2018, 14, 9693-9701.

33 M. Muthukumar, Macromolecules, 2017, 50, 9528-9560.

34 F. Horkay, I. Tasaki and P. J. Basser, Biomacromolecules, 2001, 2, 195-199.

35 T. Tanaka, D. Fillmore, S.-T. Sun, I. Nishio, G. Swislow and A. Shah, Phys. Rev. Lett., 1980, 45, 1636-1639.

36 G. S. Manning, J. Chem. Phys., 1969, 51, 924-933.

37 T. Tominaga, V. R. Tirumala, E. K. Lin, J. P. Gong, H. Furukawa, Y. Osada and W.-L Wu, Polymer, 2007, 48, 7449-7454.

$38 \mathrm{H}$. Itagaki, T. Kurokawa, H. Furukawa, T. Nakajima, Y. Katsumoto and J. P. Gong, Macromolecules, 2010, 43, 94959500.
39 S. P. Obukhov, M. Rubinstein and R. H. Colby, Macromolecules, 1994, 27, 3191-3198.

40 T. Sakai, M. Kurakazu, Y. Akagi, M. Shibayama and U. Chung, Soft Matter, 2012, 8, 2730-2736.

41 M. Rubinstein and R. H. Colby, Polymer Physics, Oxford University Press, New York, 2003.

42 J. Li, Y. Hu, J. J. Vlassak and Z. Suo, Soft Matter, 2012, 8, 81218128.

43 H. Guo, T. Kurokawa, M. Takahata, W. Hong, Y. Katsuyama, F. Luo, J. Ahmed, T. Nakajima, T. Nonoyama and J. P. Gong, Macromolecules, 2016, 49, 3100-3108.

44 Y. S. Kim, M. Liu, Y. Ishida, Y. Ebina, M. Osada, T. Sasaki, T. Hikima, M. Takata and T. Aida, Nat. Mater., 2015, 14, 10021007. 